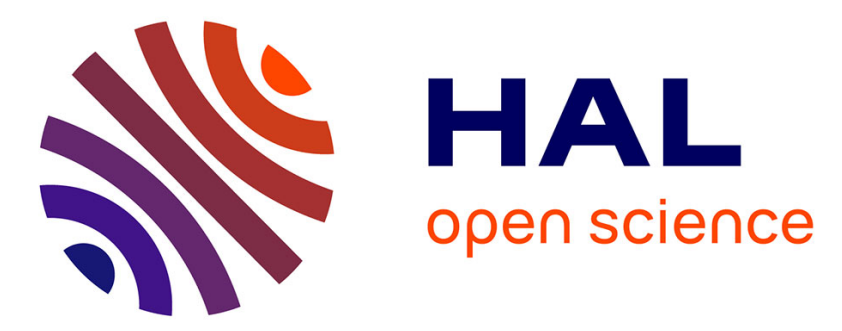

\title{
Multiscale displacement field measurement using digital image correlation: Application to the identification of elastic properties
}

Jean-Charles Passieux, Florian Bugarin, Christoph David, Jean-Noël Périé, Laurent Robert

\section{To cite this version:}

Jean-Charles Passieux, Florian Bugarin, Christoph David, Jean-Noël Périé, Laurent Robert. Multiscale displacement field measurement using digital image correlation: Application to the identification of elastic properties. Experimental Mechanics, 2015, 55 (1), pp.121-137. 10.1007/s11340-014-9872-4 . hal-00949038

\section{HAL Id: hal-00949038 \\ https://hal.science/hal-00949038}

Submitted on 19 Feb 2014

HAL is a multi-disciplinary open access archive for the deposit and dissemination of scientific research documents, whether they are published or not. The documents may come from teaching and research institutions in France or abroad, or from public or private research centers.
L'archive ouverte pluridisciplinaire $\mathbf{H A L}$, est destinée au dépôt et à la diffusion de documents scientifiques de niveau recherche, publiés ou non, émanant des établissements d'enseignement et de recherche français ou étrangers, des laboratoires publics ou privés. 


\title{
Multiscale displacement field measurement using digital image correlation:
}

\section{Application to the identification of elastic properties}

\author{
Jean-Charles Passieux • Florian Bugarin • \\ Christoph David · Jean-Noël Périé .
}

Laurent Robert

Current draft version: February 19, 2014

\begin{abstract}
As any Digital Image Correlation (DIC) method, Finite-Element (FE) based DIC methods lead to uncertainties which are related to the spatial resolution (in pixel / element). To overcome the tricky and well-known compromise between spatial resolution and uncertainty, a multiscale approach to FE-DIC is proposed. Additional nearfield images are used to improve locally the resolution of the measurement for a given measurement mesh. An automatic and accurate estimation of the nearfield / farfield transformation is obtained by a dedicated DIC based method, in order to bridge precisely the measurement performed at both scales. This multiscale measurement is then associated to a multiscale Finite Université de Toulouse; ICA (Institut Clément Ader), INSA/UPS/Mines Albi/ISAE, 135 avenue de Rangueil F-31077 Toulouse, France

J.-C. Passieux

E-mail: passieux@insa-toulouse.fr

F. Bugarin

E-mail: bugarin@mines-albi.fr

C. David

E-mail: chdavid@mines-albi.fr

J.-N. Périé

E-mail: jean-noel.perie@iut-tlse3.fr

L. Robert

E-mail: laurent.robert@mines-albi.fr
\end{abstract}


Element Model Updating (FEMU) identification technique. After being validated on synthetic test cases, the method is applied to a tensile test carried out on an open-hole specimen made of glass / epoxy laminate. The four in-plane orthotropic elastic parameters are identified at different levels of loading. Results show that the multiscale approach greatly improves the uncertainty of both the measured displacements and the identified material parameters.

Keywords multiscale $\cdot$ mechanical identification $\cdot$ full-field measurement $\cdot$ digital image correlation $\cdot$ finite element

\section{Introduction}

Over the three last decades, full-field measurement techniques have become increasingly popular in the community of experimental mechanics. One can report geometrical methods, such as grid method, moiré, or Digital Image Correlation (DIC), and interferometric methods such as electronic speckle pattern interferometry, holographic interferometry, or shearography, etc. [1]. Among these experimental techniques, DIC is probably the most used either in the academic or industrial community thanks to its (apparent) simplicity [2]. Displacement (or strain) fields measured by DIC can also be used for constitutive parameters identification, e.g. from heterogeneous mechanical tests. In fact, with conventional identification methods $[3,4]$ more than one test is usually required for the identification of the constitutive parameters. Since they provide a sufficiently large amount of information, full-field measurement techniques allow the identification of several parameters from a single non-homogeneous test [5-10]. For that purpose, several identification strategies based on full-field measurements have been recently developed, see e.g. [8] or [1] for more details. However a drawback of this approach is obviously that the level of uncertainty associated with the identified parameters depends on the quality of the kinematic measurements [11], and thus in our concern, of the DIC displacement measurement uncertainties [12]. The latter are related at least to the DIC method itself [13], but also depend strongly on the spatial resolution of the displacement measurement $[14,13]$.

DIC is based on the assumption that the distortion of the image pattern is due to the mechanical transformation of the seen object. Classically, DIC meth- 
ods consist in the minimization of a quantity that express the difference between the gray levels in the undeformed $\mathcal{I}_{0}$ and the deformed $\mathcal{I}_{1}$ images. DIC methods roughly comprise two broad categories: (i) subset-based method (or local approach) in which the parameters of a shape function (typically linear or quadratic) are searched across a (generally small and square) subset of the whole region of interest (ROI) [15], and (ii) the global approach, that minimize the criteria over the entire ROI at one time. In this work, a global DIC method based on Finite Element kinematics (FE-DIC) is used, following [16-18]. The main advantage, besides the fact that between the numerical simulations and the full-field measurements no projection is necessary because the displacement is discretized in the same way, is that FE-DIC approaches reduce measurement uncertainties because they require the continuity of the found displacement field throughout the ROI [13]. In the following, a unique mesh is used for the discretization of both experimental and simulated displacements.

Concerning the measurement uncertainties in DIC, the most restricting ones are the random errors, which are linked to the subset size (local approach) $[19,12$, 2 ] or to the number of pixels per element (global approach) [17], thus defining the spatial resolution (expressed in pixel). More precisely, the higher the number of pixels per element is, the smaller uncertainties are. This compromise is classical to DIC $[17,2]$. It is worth noting that a kinematic model (i.e. mesh) sufficiently rich to catch strain gradients, may lead to high spatial resolution, to the detrimental of larger measurement errors.

On the other hand, if one wishes to identify all the model parameters, the kinematic field is obviously not enough, and the static quantities must also be taken into account [10]. To this purpose, the region of interest (ROI) must include the boundaries of the domain on which a resultant of the external loads is partially measured. The image definition which is a characteristic of the camera (number of pixels) and the size of the ROI, which characterizes the experimental test, set the image resolution (in pixel / $\mathrm{mm}$ ). As a result, the spatial resolution is thus a highly constrained experimental parameter (large structure, high gradients, complex geometry, low levels of deformation in the elastic range, etc.). In some cases it may be that the measurement uncertainties become disadvantageous for the inverse parameter identification [20]. This is particularly true when it comes 
to identifying the model parameters describing the elastic behavior of composite materials.

To overcome this spatial resolution / uncertainty compromise, we introduce in this work a nearfield / farfield multiscale approach, that utilizes a Finite Elementbased DIC measurement method and a Finite Element Model Updating (FEMU) identification procedure $[21,22]$. In a first step, we propose to use two cameras that acquire images with two different image resolutions to measure the displacement fields by FE-DIC on the surface of the specimen. A series of images capture the full specimen (farfield images: at the scale of the structure), while a second series of images zoom on a structural detail (nearfield images: for example, in a local region where the displacement field is particularly sensitive to the parameters to be identified). The FE simulation mesh is used for the FE-DIC measurement at both scales. An image registration process that automatically and accurately repositions the nearfield image into the farfield image, based on a global DIC approach, allows to place precisely the mesh on the nearfield image.

In a second step, an ad hoc inverse multiscale identification method is presented. Based on the FEMU, it takes advantage of the multiscale FE-DIC. On the one hand the farfield FE-DIC measurement provides representative Dirichlet boundary conditions for the numerical simulation $[9,23,10]$. The corresponding reaction force is compared to the one provided by a load cell. The addition of this force term in the cost function is essential for the identification of elastic moduli. On the second hand, the nearfield FE-DIC measurement provides a high spatial resolution kinematics field for the test / calculation displacement comparison, in a region where the model parameters are particularly sensitive.

The outline of the paper is as follows: in Section 2, after a brief review on digital image correlation, an automatic nearfield / farfield image registration technique is proposed. An a priori analysis of the multiscale FE-DIC approach is then presented in Section 3. Measurement uncertainties are evaluated through synthetic images (shifted or strained). In Section 4, the method is applied to an open-hole tensile test performed on a glass/epoxy laminate. The experimental set-up is presented and both farfield and nearfield images are analyzed. In section 5, after being validated on previous synthetic test cases, the multiscale inverse method is applied to identify in-plane parameters of an orthotropic elastic model. 


\section{Multiscale digital image correlation}

\subsection{Digital Image Correlation}

Digital Image Correlation (DIC [24,14]) consists in seeking the displacement field $\mathbf{u}$ that register an image $\mathcal{I}_{1}$ into another image $\mathcal{I}_{0}$ of a specimen in two different loading conditions. Following [17,13], a weak form of the gray level conservation equation [25] is written globally over the whole region of interest (ROI):

$$
\mathbf{u}^{\star}=\underset{\mathbf{u}(\mathbf{x})}{\arg \min } \int_{R O I}\left(\mathcal{I}_{0}(\mathbf{x})-\mathcal{I}_{1}(\mathbf{x}+\mathbf{u}(\mathbf{x}))\right)^{2} d \mathbf{x}
$$

In practice, the unknown displacement field $\mathbf{u}: \mathcal{I}_{0} \rightarrow \mathcal{I}_{1}$ is sought in an approximation subspace $\mathcal{U}^{N}$, spanned by a finite dimension interpolation basis $\phi_{i}(\mathbf{x})$ as follows:

$$
\mathbf{u}=\sum_{i=1}^{N} \phi_{i}(\mathbf{x}) q_{i}
$$

where $\mathbf{q}$ is the corresponding vector of degrees of freedom $q_{i}$. A large choice of interpolations can be used in this framework, among which Fourier series [26,27], B-Splines [28,29], separation of variables [30], mechanical based analytical functions $[31,32]$ or precomputed numerical functions [20]. In this work, a DIC method based on Finite Element kinematics (FE-DIC) has been developed following [1618]. The stationarity conditions associated to the minimization of the linearized problem (1) yields a set of linear systems:

$$
\mathbf{M} \mathbf{q}^{k}=\mathbf{b}^{k}
$$

where $\mathbf{M}$ is a $N \times N$ matrix called the correlation operator and $\mathbf{b}$ the corresponding right-hand-side:

$$
\begin{aligned}
\mathbf{M}_{i j} & =\int_{R O I}\left(\nabla \mathcal{I}_{0}^{\top} \phi_{i}\right)\left(\nabla \mathcal{I}_{0}^{\top} \phi_{j}\right) d \mathbf{x} \\
\mathbf{b}_{i}^{k} & =\int_{R O I}\left(\nabla \mathcal{I}_{0}^{\top} \phi_{i}\right)\left(\mathcal{I}_{0}(\mathbf{x})-\mathcal{I}_{1}\left(\mathbf{x}+\mathbf{u}^{k-1}\right)\right) d \mathbf{x}
\end{aligned}
$$

where $\mathbf{u}^{k-1}=\sum_{j=0}^{k-1} \sum_{i=1}^{N} \phi_{i} q_{i}^{j}$ is the approximation of the displacement at iteration $k-1$. Since $\mathbf{x}+\mathbf{u}^{k-1}$ may be non-integer, a gray level interpolation is required to evaluate the right-hand-side. In this paper, a classical spline interpolation is used. 
The definition of the approximation subspace $\mathcal{U}^{N}$ has a direct impact on the accuracy of the estimation. First $N$ should be far lower than the number of pixels in the ROI because of the ill-posedness nature of the correlation problem. Namely, the larger $N$, the larger are measurement uncertainties. However, $\mathcal{U}^{N}$ should be rich enough to accurately represent the a priori unknown displacement. Namely, measurement uncertainties result from a compromise between the accuracy of the displacement interpolation, that manages what is called the "mismatch error" in Bornert et al. [12], and the so-called "ultimate error" when the displacement interpolation is sufficiently accurate according to the true deformation of the image. In the case of FE-DIC, a mesh that would be optimal for simulation purposes, is not necessarily optimal for the DIC measurement. Thus the choice of a common mesh is not, in general, an easy task in the context of identification [20].

Most often, digital images are taken at one single resolution. In this case, a first way to use a simulation mesh including small elements (i.e. with a poor measurement resolution) is to search only for solutions that have some numerical [30] or mechanical [20,33] regularity. The aim of this article is to explore another route, which consists in using images of the same speckle at more than one resolution. The multiscale (or multi-resolution) measurement technique proposed herein, is designed to adapt the image resolution to the mesh and not the reverse.

In this paper, the case of image pairs taken from two cameras at two different scales is considered, one being denoted farfield and the second nearfield. The first question that arises, is how to characterize accurately the transformation which links the position of a point in the nearfield image to its corresponding in the farfield image. For this purpose, a dedicated digital image correlation technique is devised in section 2.2 .

\subsection{Farfield / nearfield image registration}

A key issue of the proposed multiscale correlation method consists in registering accurately the nearfield reference image $\mathcal{I}_{0}^{n}$ in the farfield reference image $\mathcal{I}_{0}^{f}$. In 
this study, this multiscale transformation $\mathbf{t}(\mathbf{x})$ is estimated by considering only the speckle of both scales. Thus a dedicated digital image correlation algorithm is devised for that purpose. Indeed, $\mathbf{t}(\mathbf{x})$ is sought to minimize the gray level conservation equation:

$$
\mathbf{t}^{\star}=\underset{\mathbf{t}(\mathbf{x})}{\arg \min } \int_{R O I}\left(\mathcal{I}_{0}^{n}(\mathbf{x})-\mathcal{I}_{0}^{f}(\mathbf{x}+\mathbf{t}(\mathbf{x}))\right)^{2} d \mathbf{x}
$$

where the ROI corresponds, here, to the entire nearfield image $\mathcal{I}_{0}^{n}$.

As mentionned previously, the key point of a correlation method is to propose an adequate and sufficiently accurate kinematic interpolation model in the DIC algorithm. One way to do this is to use the a priori knowledge of the unknown transformation in order to reduce the number of unknowns $N$. In the context of the near/farfield registration, and since the studied specimen are assumed to be planar, the proposed contribution consists in seeking the transformation $\mathbf{t}$ as an arbitrary homography $\mathbf{H}$ relating $\mathcal{I}_{0}^{n}$ to $\mathcal{I}_{0}^{f}$. An homography is an invertible mapping of points (and lines) on the projective plane $\mathbb{P}^{2}$, represented by a non-singular $3 \times 3$ matrix $\mathbf{H}$. In other words, for a given point $\mathbf{x} \in \mathcal{I}_{0}^{n}$ and its corresponding point $\mathbf{x}_{i}^{\prime} \in \mathcal{I}_{0}^{f}$ we have the constraint: $\mathbf{x}^{\prime}=\mathbf{H} \mathbf{x}$. Note that $\mathbf{H}$ can be multiplied by an arbitrary non-zero constant without modifying the projective transformation. Thus $\mathbf{H}$ is an homogeneous matrix with only 8 degrees of freedom even though it contains 9 parameters. So, the dimension of the approximation subspace is reduced to 8 for the whole nearfield image. Finally, the solution is computed by a Levenberg-Marquardt algorithm applied to the following problem:

$$
\mathbf{H}^{\star}=\underset{\mathbf{H} \in \mathcal{M}_{3,3}(\mathbb{R})}{\arg \min } \int_{R O I}\left(\mathcal{I}_{0}^{n}(\mathbf{x})-\mathcal{I}_{0}^{f}(\mathbf{H} \mathbf{x})\right)^{2} d \mathbf{x}
$$

where the numerical integration over the ROI $\mathcal{I}_{0}^{n}$ is performed by a mid-pixel rectangle method following [13].

Since the scales can be very different between near and farfield images, this algorithm has to be initialized with a coarse approximation of the homography. Typically, an homography is estimated between two images by finding a set of $r$ matched points $\left(\mathbf{m}_{i}, \mathbf{m}_{i}^{\prime}\right)$. Three algorithms have been compared for extracting and matching interest points : SURF [34], MSER [35], SIFT [36]. In most of the examples that have been processed, the SIFT algorithm [36] was the most efficient because it provides a large sets of matched points for most of our configurations. 
Next, consider a sufficient set (i.e. $r \geq 8$ ) of matched points $\left(\mathbf{m}_{i}, \mathbf{m}_{i}^{\prime}\right)$. Written element by element, in homogenous coordinates one gets the following constraint:

$$
\left(\begin{array}{c}
x_{i}^{\prime} \\
y_{i}^{\prime} \\
z_{i}^{\prime}
\end{array}\right)=\left(\begin{array}{lll}
h_{11} & h_{12} & h_{13} \\
h_{21} & h_{22} & h_{23} \\
h_{31} & h_{32} & h_{33}
\end{array}\right)\left(\begin{array}{c}
x_{i} \\
y_{i} \\
z_{i}
\end{array}\right) \Leftrightarrow \mathbf{m}_{i}^{\prime}=\mathbf{H m}_{i}
$$

which, in inhomogenous coordinates, corresponds to:

$$
\begin{gathered}
x_{i}^{\prime} \equiv \frac{x_{i}^{\prime}}{z_{i}^{\prime}}=\frac{h_{11} x_{i}+h_{12} y_{i}+h_{13} z_{i}}{h_{31} x_{i}+h_{32} y_{i}+h_{33} z_{i}} \\
y_{i}^{\prime} \equiv \frac{y_{i}^{\prime}}{z_{i}^{\prime}}=\frac{h_{21} x_{i}+h_{22} y_{i}+h_{23} z_{i}}{h_{31} x_{i}+h_{32} y_{i}+h_{33} z_{i}}
\end{gathered}
$$

Without loss of generality, $z_{i}$ is set to $z_{i}=1$ and (7) and (8) are rearranged in order to have an overdetermined linear system (solved in a least square sense) where coefficients of $\mathbf{H}$ appear linearly:

$$
A h=0 \quad \text { where } \quad A=\left(\begin{array}{lllll}
a_{x 1} & a_{y 2} & \ldots & a_{x r} & a_{y r}
\end{array}\right)^{\top}
$$

and

$$
\begin{aligned}
& a_{x i}=\left(\begin{array}{llllllll}
-x_{i}-y_{i} & -1 & 0 & 0 & 0 & x_{i}^{\prime} x_{i} & x_{i}^{\prime} y_{i} & x_{i}^{\prime}
\end{array}\right)^{\top} \\
& a_{y i}=\left(\begin{array}{llllllll}
0 & 0 & 0 & -x_{i}-y_{i} & -1 & y_{i}^{\prime} x_{i} & y_{i}^{\prime} y_{i} & y_{i}^{\prime}
\end{array}\right)^{\top} \\
& h=\left(\begin{array}{lllllllll}
h_{11} & h_{12} & h_{13} & h_{21} & h_{22} & h_{23} & h_{31} & h_{32} & h_{33}
\end{array}\right)^{\top}
\end{aligned}
$$

This two step method is applied to synthetic images whose construction is detailed in section 3.1. In order to visualize the results, a point cloud which corresponds to the nodes of a FE mesh are adjusted on the nearfield image $\mathcal{I}_{0}^{n}$. Their image under the initial and optimized homography operators are plotted on the farfield image $\mathcal{I}_{0}^{f}$ in Figure 1. (Note that the homography is estimated in the nearfield region only and extrapolated in the farfield). The corresponding raw discrepancy maps (in gray levels for a 256 gray levels range) $\mathcal{I}_{0}^{n}\left(\mathbf{x}_{i}\right)-\mathcal{I}_{0}^{f}\left(\mathbf{H x}_{i}\right)$ are plotted in Figure 2. Theoretically, with exact matched points the result of the initial value of $\mathbf{H}$ estimated by SIFT should be accurate. But, in practice, the couples $\left(\mathbf{m}_{i}, \mathbf{m}_{i}^{\prime}\right)$ are not properly matched and the solution of (9) is inaccurate, see Figure 1(left). The gray level conservation is also poorly verified since the standard 

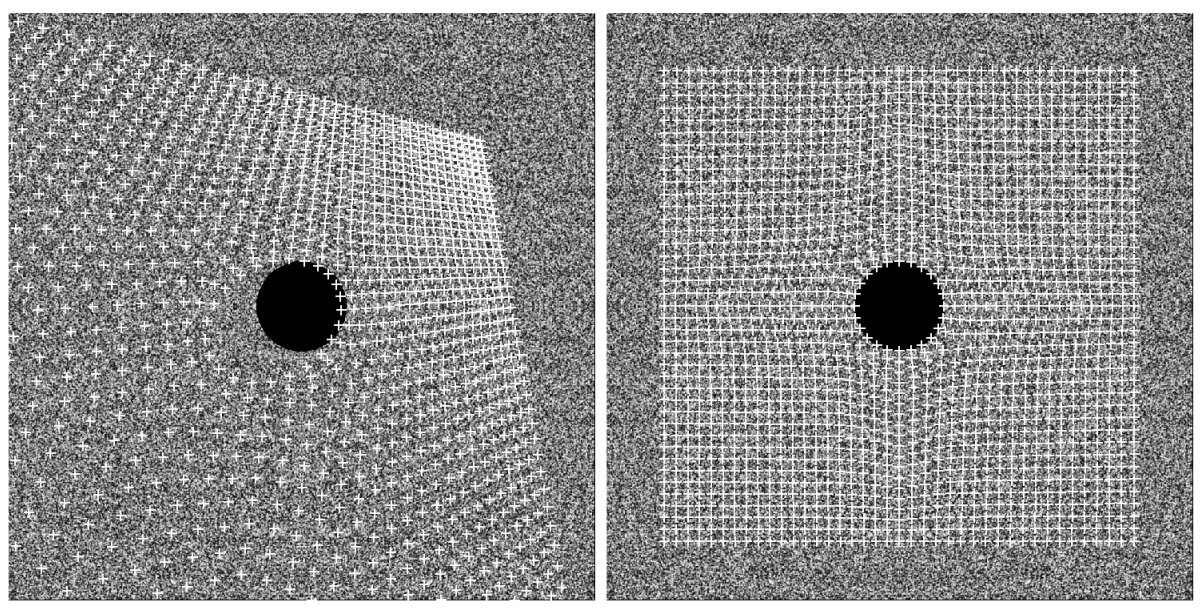

Fig. 1 The mesh nodes are adjusted on the nearfield reference image $\mathcal{I}_{0}^{n}$ by hand. The images of these nodes under the initial homography estimated by SIFT (left) and the one measured by the DIC technique (right) are plotted on the farfield reference image $\mathcal{I}_{0}^{f}$.
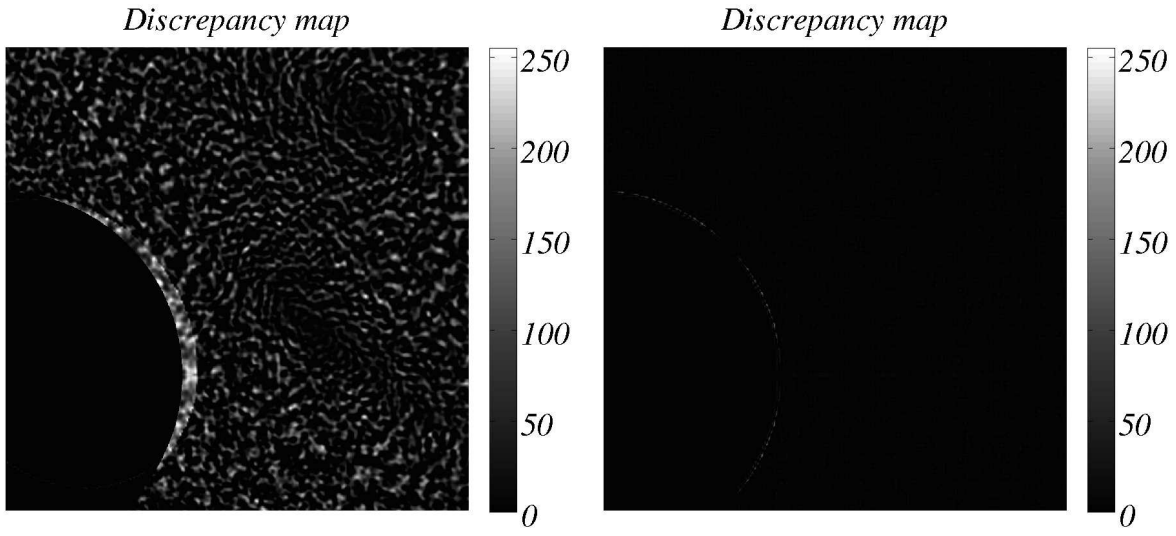

Fig. 2 The raw discrepancy map $\mathcal{I}_{0}^{n}\left(\mathbf{x}_{i}\right)-\mathcal{I}_{0}^{f}\left(\mathbf{H x}_{i}\right)$ (in gray levels) with the initial homography estimated by SIFT (left) and the one measured by the DIC technique (right)

deviation of the residual map is equal to $23.5 \%$ of the dynamic range of the image, see Figure 2(left). However it provides a sufficiently good approximation to initialize the DIC algorithm of equation (5), see Figure 1(right). The latter provides a very good estimation of the transformation since the gray level conservation seems accurately verified: standard deviation of the discrepancy map is equal to $1.14 \%$ of the dynamic range, as shown in Figure 2(right). 
Remark. In the particular case of the synthetic images described in 3.1, the homography has additional properties. First, because the scales are the same everywhere in $\mathcal{I}_{0}^{n}$, the coefficients $h_{31}$ and $h_{32}$, responsible of the non-linearity in (7) and (8), are equal to zero.

$$
h_{31}=h_{32}=0
$$

In such a condition, the homography is said affine. Second, because, in this case, $\mathbf{H}$ is a composition of a pure scaling of factor 5 and a translation, the following relations must apply:

$$
\begin{aligned}
& h_{12}=h_{21}=0 \\
& h_{11}=h_{22}=\frac{1}{5} .
\end{aligned}
$$

Finally, $h_{13}$ and $h_{23}$, coefficients of the rigid body translations, are arbitrary and only depend on the localization of the nearfield region of interest. Thus, in addition to the measurement of the discrepancy map of Figure 2, a good way to validate the proposed DIC algorithm is to underline that the optimized homography (13)

$$
\mathbf{H}^{\star}=\left(\begin{array}{ccc}
0.199899 & 5.8610^{-6} & 499.812 \\
-3.4110^{-5} & 0.199985 & 359.793 \\
-1.2210^{-7} & -1.1110^{-10} & 1
\end{array}\right)
$$

solution of (5), meets properties (10), (11) and (12).

\section{A priori analysis of synthetic images}

Images used in these sections are synthetized from a mechanical analytical displacement field. Their construction is detailed in section 3.1. The nearfield / farfield registration described in the previous section is performed. It is then possible to quantify both ultimate and model random errors with the same meshes in section 3.2 .

\subsection{Multiscale image synthesis}

The main idea is to build a set of synthetic images in order to evaluate the deviation between the measured and prescribed displacement fields. The set of synthetic 
speckle-pattern images is obtained using the TexGen software [37]. This software has been developed to produce synthetic speckle-pattern images which simulate real DIC speckle patterns as realistically as possible. Deformed synthetic images can also be generated with any displacement field.

Details of the speckle-pattern generator algorithm can be found in [37]. Let us simply mention that Perlin's coherent noise function [38] is used to generate a continuous texture function $\eta$ :

$$
\begin{aligned}
\eta:[-1,1]^{2} & \rightarrow[0,1] \\
(x, y) & \rightarrow \eta(x, y) .
\end{aligned}
$$

The speckle-pattern image is generated by a photometric mapping and an 8-bit digitization of the texture function computed for each integer pixel of the image. The integration of the texture function over the domain corresponding to the photosensitive area of one pixel is performed by a super-sampling technique in order to simulate the pixel fill factor. A reference speckle-pattern image, represented by a gray level function $\mathcal{I}_{0}(\mathbf{x})$, is first generated. Next, the deformed speckle-pattern image $\mathcal{I}_{1}(\mathbf{x})$ is generated by applying a transformation $\Phi$ using the optical flow conservation equation:

$$
\mathcal{I}_{1}(\mathbf{x})=\mathcal{I}_{0}\left(\Phi^{-1}(\mathbf{x})\right), \quad \text { with } \quad \Phi(\mathbf{x})=\mathbf{x}+\mathbf{u}(\mathbf{x})
$$

where $\mathbf{u}$ must be an analytical $\mathcal{C}^{1}$ function in order to ensure the computation of $\Phi^{-1}$ thanks to an iterative root finding algorithm. Note that $\Phi$ is applied to the continuous texture function $\eta$, and not to the pixel (i.e. discrete gray level) values of $\mathcal{I}_{0}$. Then, the continuous deformed texture obtained by solving (14) is mapped to generate the deformed image. Regarding classical procedures (e.g. based on gray level interpolation in the space [39] or Fourier [40] domain), this method is known to limit the introduction of any bias due to interpolation.

In order to perform a virtual mechanical test, the displacement $\mathbf{u}$ is calculated from the analytical solution $\mathbf{u}^{L}$ of an infinite orthotropic open hole plate in vertical remote tension. Theoretical solution of this problem has been proposed in [41] and already used for identification purposes in [6]. The four orthotropic parameters are set to $E_{l}=60 \mathrm{GPa}, E_{t}=56 \mathrm{GPa}, G_{l t}=4.26 \mathrm{GPa}$ and $\nu_{l t}=0.049$, the hole radius is set to $r=2 \mathrm{~mm}$ and the prescribed stress to $\sigma_{\infty}=100 \mathrm{MPa}$. 


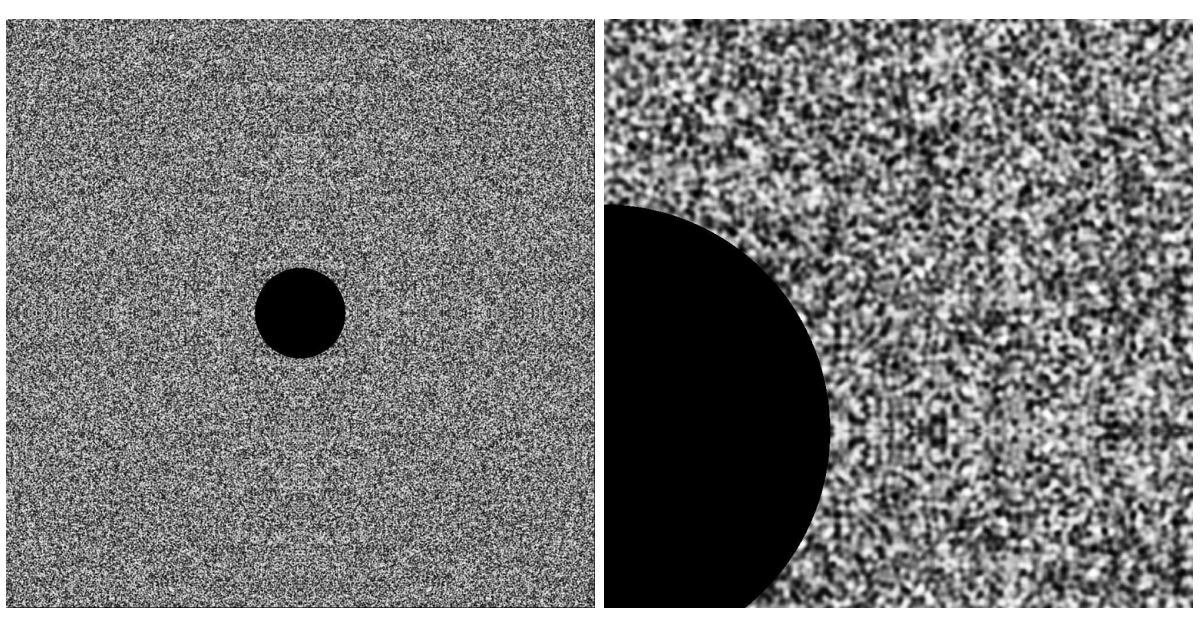

Fig. 3 Reference image generated with TexGen for the farfield image $\mathcal{I}_{0}^{f}$ (left) and the nearfield image $\mathcal{I}_{0}^{n}$ (right)

A first pair of $1000 \times 1000$ pixels images $\left(\mathcal{I}_{0}^{f}, \mathcal{I}_{1}^{f}\right)$ is generated with a resolution of $26 \mu \mathrm{m}$ per pixel. This pair of images correspond to the farfield, as the field of view is $26 \mathrm{~mm}$ wide. A second pair of $1000 \times 1000$ pixels images $\left(\mathcal{I}_{0}^{n}, \mathcal{I}_{1}^{n}\right)$ is generated, but with a resolution of $5.2 \mu \mathrm{m}$ per pixel ( 5 times smaller). This one corresponds to the nearfield images, with a field of view of $5.2 \mathrm{~mm}$ wide. The caracteristic size of the speckle is $34 \mu \mathrm{m}$, which corresponds to 1.3 pixel in the farfield images and 6.5 pixel in the nearfield images. These speckles, shown in Figure 3, are thus respectively suboptimal and superoptimal according to [2]. The corresponding theoretical reference nearfield and farfield displacement fields are shown in Figure 4.

\subsection{Separate analysis of multiresolution images}

As mentioned above, in DIC, the total measurement uncertainties is classically viewed as a competition of the so-called ultimate and model errors [12,29]. Knowing the exact analytical displacement field $\mathbf{u}_{r e f}$ (see section 3.1), it possible to compute the random error of a displacement field $\mathbf{u}$ measured by FE-DIC as the standard deviation of the discrepancy $\Delta \mathbf{u}=\mathbf{u}-\mathbf{u}_{\text {ref }}$ over the pixels overlaped by finite elements within the ROI. The discrepancy term $\Delta \mathbf{u}$ depends on the chosen uncertainty: 

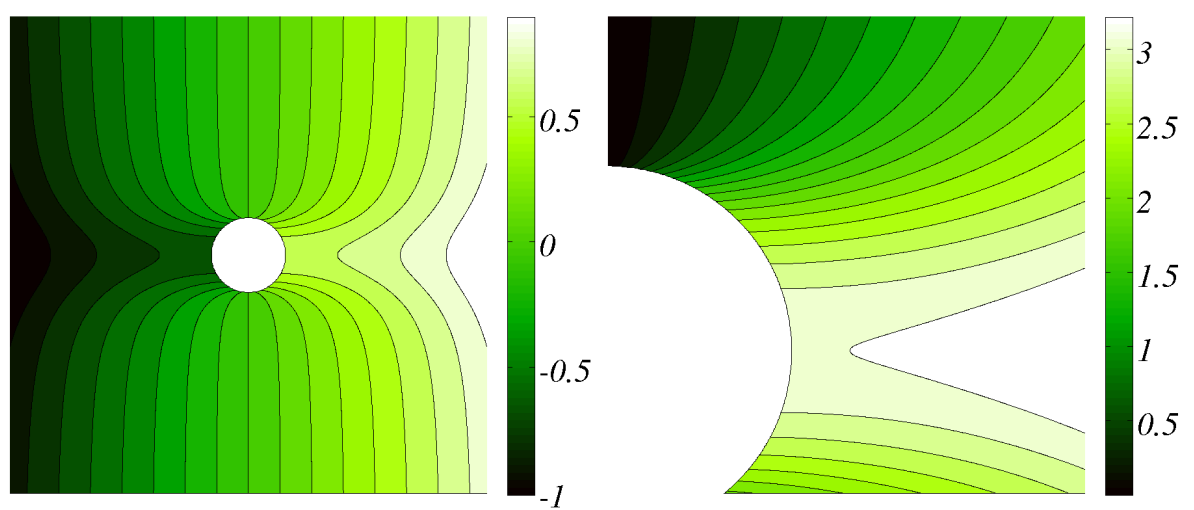

Fig. 4 Horizontal $x$-component of the reference displacement field in pixel, in farfield (left) and in nearfield (right)

- ultimate error. A simple rigid body translation along $x$-axis is imposed to the reference image, here by a shift in the Fourier space $[17,12]$. Such a displacement field does belong to the finite element approximation subspace $\mathcal{U}^{h}$. Thus the ultimate error only considers the errors inherent to the DIC technique in situation where the adopted kinematic model of the DIC algorithm perfectly fits the actual displacement field in the image. In this work, the magnitude of the $x$-component of the displacement is set to $\mathbf{u}_{r e f}^{\text {shift }}=0.5$ pixel since it maximises the standard uncertainty in the case of noiseless images [42]. The FE-DIC measurement yields an inexact displacement map $\mathbf{u}_{m}$ which is used to estimate the ultimate random error as follows:

$$
\sigma^{u l t}=\sigma\left(\mathbf{u}_{m}-\mathbf{u}_{r e f}^{\text {shift }}\right)
$$

where $\sigma(\cdot)$ is the standard deviation operator.

- model error. It correponds to the so-called interpolation error in the computational mechanics jargon [29]. It only consists in the evaluation of the distance between a non-constant analytical displacement field and its projection on the finite element approximation subspace $\mathcal{U}^{h}$. No DIC is performed at this stage. In this paper, the mechanical analytical displacement field described in the previous section serves as the reference $\mathbf{u}_{r e f}^{L}$. Its projection $\mathbf{u}_{p r o j}$ on the FE approximation subspace is computed in the least square sense:

$$
\mathbf{u}_{\text {proj }}=\underset{\mathbf{u} \in \mathcal{U}^{h}}{\arg \min } \sum_{i=1}^{m}\left(\mathbf{u}_{r e f}^{L}-\mathbf{u}\right)^{2}
$$


which only requires the resolution of a linear system whose operator is the finite element mass matrix. The model error is thus estimated by:

$$
\sigma^{\text {mod }}=\sigma\left(\mathbf{u}_{\text {proj }}-\mathbf{u}_{r e f}^{L}\right)
$$

- total error. The mechanical analytical field $\mathbf{u}_{r e f}^{L}$ is prescribed to the reference image as described in section 3.1. The measured displacement $\mathbf{u}_{m}$ between these synthetic images is computed by performing a FE-DIC. The total error is then computed as:

$$
\sigma^{t o t}=\sigma\left(\mathbf{u}_{m}-\mathbf{u}_{r e f}^{L}\right)
$$

This quantity, which measures the exact error between the measured and reference displacement maps, takes into account both sources of uncertainties.

Remark. The total error is always greater than the model error $\sigma^{\text {tot }} \geq \sigma^{\text {mod }}$. However, the ultimate error may, in some cases, be slightly lower than the total error since a prescribed rigid body translation of 0.5 pixel does not always maximize the ultimate error, in particular when noise is present in the image, or when the characteristic speckle size is not optimal [42].

This a priori performance analysis is performed with both fields of view as a function of the characteristic mesh size (which corresponds to the spatial resolution i.e. the subset size for subset-based DIC approaches). Therefore, a set of eleven unstructured finite element meshes are generated with Gmsh [43]. Their elements size are rather tightly clustered around the mean value that ranges from $78 \mu \mathrm{m}$ to $5 \mathrm{~mm}$, as shown in Figure 5. The mesh is adjusted on the farfield image and transfered to the nearfield image thanks to the inverse of the optimized homography $\left(\mathbf{H}^{\star}\right)^{-1}$. Like this, the same meshes are used for both near and farfield images analyses.

Figure 6 presents the evolution of ultimate, model and total random errors in millimeter as a function of the element size in millimeter, for both nearfield (in red) and farfield (in black) images. When nearfield and farfield analyses are considered independantly, it can be observed that the larger the elements (or equivalent, the more pixels per element), the lower is the ultimate error. Conversely, the larger the elements, the higher is the model error. The overmentionned compromise can be seen graphically on this figure, since the total error results from the competition of these two antithetical behaviors. 

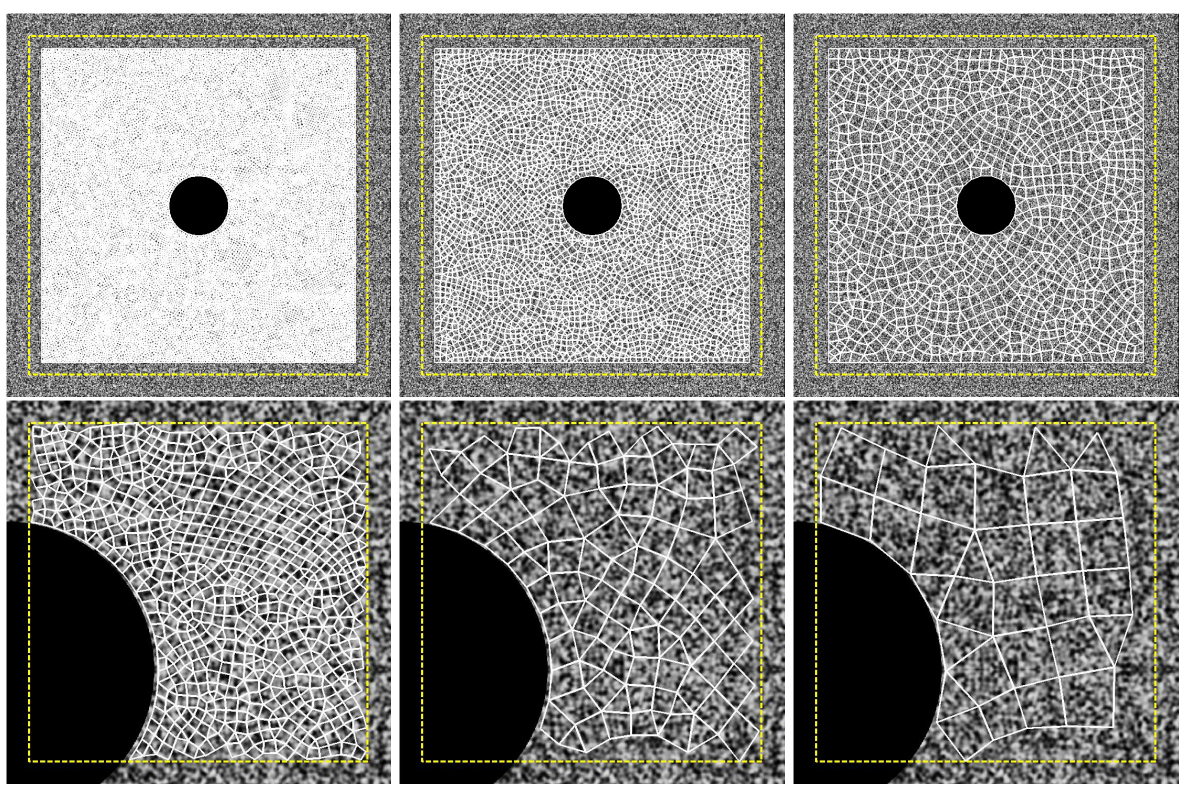

Fig. 5 Example of unstructured meshes used for the a priori uncertainty analysis described in this section: from left to right, the average element size is respectively 163,411 and $719 \mu \mathrm{m}$, which corresponds to 6,16 and 28 pixels per element width in the farfield image (top) and 31, 79 and 138 pixels per element width in nearfield image (bottom).

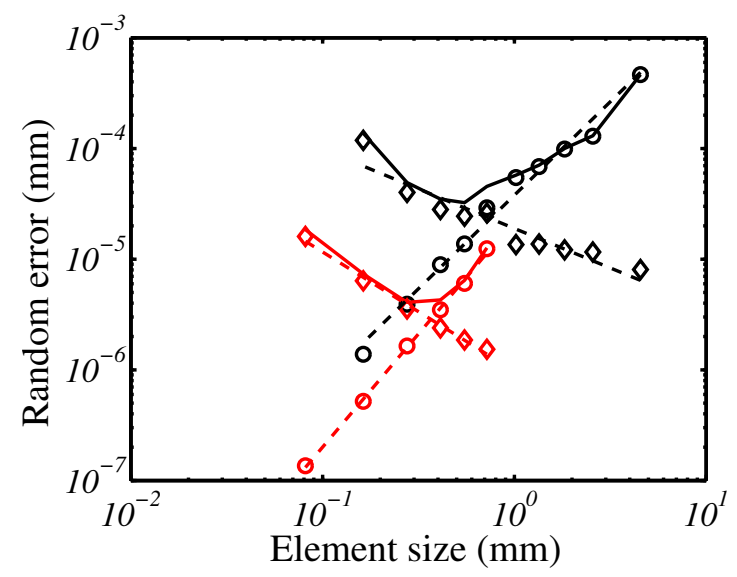

$-\diamond$ - ultimate error (farfield)

- $\boldsymbol{\theta}$ - model error (farfield)

— total error (farfield)

$-\diamond$ - ultimate error (nearfield)

- $\bullet$ - model error (nearfield)

— total error (nearfield)

Fig. 6 Evolution of the ultimate (wedge), model (circles) and total (solid line) random errors as a function of the element size in millimeter for both nearfield (in red) and farfield images (in black). 
When nearfield and farfield curves are compared, it appears that the model errors seems to broadly follow the same trend. Theoretically, they should be aligned, since this error simply depends on the physical mesh size $(\mathrm{mm})$. In practice, it is not exactly the case, since the number of elements considered for computing this error is not the same in nearfield and farfield analyses as shown in Figure 5. Conversely, the ultimate error associated to the nearfield image is much lower than that of the farfield, for a given element size. This gain can be explained, almost in its entirety, by the resolution ratio. As a result, the total error is logically shifted by the same ratio, along the direction of the model error.

A naive conclusion would be to use exclusively high definition images everywhere on the specimen. But, it is neither conceptually desirable nor technically possible for the following reasons:

- even if ultra-high definition digital camera (up to 29 MPixels) are now available at a reasonnable price, there will always exist technical limits. The compromise between the image resolution and the ROI size will remain, since, as stated in section 5.1, a large field of view may be requested in the context of identification. For representative structures, the ratio between the structural scale and the detail scale does generally not counterbalance by the increase in camera definition.

- in addition, depending on the application, the choice of the resolution may be limited by the acquisition framerate [44-46].

- generally, the finite element meshes used for simulation are only refined where higher gradients are expected, in order to rationalise computational costs [47]. It is thus unnecessary to have the same image resolution everywhere.

- computational mechanics develop more and more multiscale models that describe the behavior at two or more different scales (homogenized/refined). Dedicated measurement techniques have to be developped concurrently.

As a conclusion, for a given physical element size, the total error is thus significantly reduced thanks to such a multiscale approach. In other words, for a given target error, the multiscale measurement makes it possible to use much smaller elements. Consequently, it makes the use of a simulation mesh for measurement purposes more flexible. 

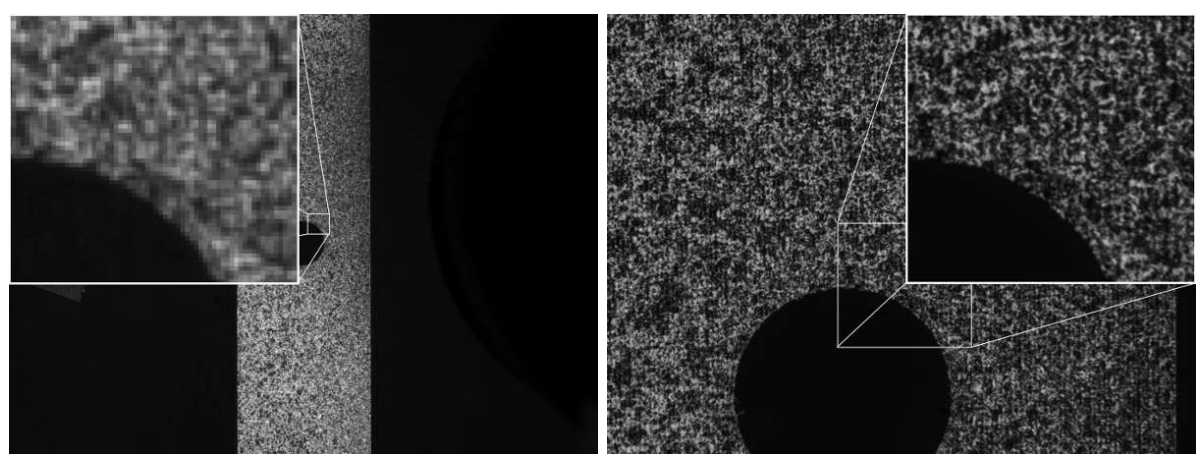

Fig. 7 Images taken at different scales. A zoom on a particular region is shown to compare the corresponding spatial resolutions

\section{Analysis of real images}

4.1 Description of the test and experimental setup

The proposed multiscale methodology is now applied to a real experiment. A quasistatic tensile test is performed on an open hole glass/epoxy composite coupon.

The base plate was manufactured by stacking four pre-preg plies made of 8-harness satin balanced woven fabric (8-HS) in the same draping direction [48]. It was formed using a vacuum bag technique and cured in a polymerization oven. The $1.26 \mathrm{~mm}$ thick laminated plate was cut with a diamond wheel into a straight-sided $30 \times 250 \mathrm{~mm}[0]_{4}$ coupon (i.e. orientated at $0^{\circ}$ with respect to the warp direction). Once the tabs glued, the gauge section is $150 \mathrm{~mm}$ long. Finally, a $10 \mathrm{~mm}$ hole is drilled in the centre of the specimen. The macroscopic behavior of the studied thin laminate is assumed to be orthotropic without in-plane bending-twisting coupling. The major material axis is aligned with the tensile direction. In the following, a $2 \mathrm{D}$ stress state is assumed. As proposed by [6,22], the idea is to take advantage of the non-uniformity of the resulting $2 \mathrm{D}$ strain field in order to identify the four in-plane elastic properties at once.

The test was carried out on an electromechanical tensile machine (Instron 5800). The loading was periodically interrupted after a load increment of approximately $0.5 \mathrm{kN}$ up to $5 \mathrm{kN}$. In between the steps, the loading rate was around $0.25 \mathrm{~mm} / \mathrm{mn}$. At each load step, once the load stabilized, both the farfield and nearfield images were recorded. 


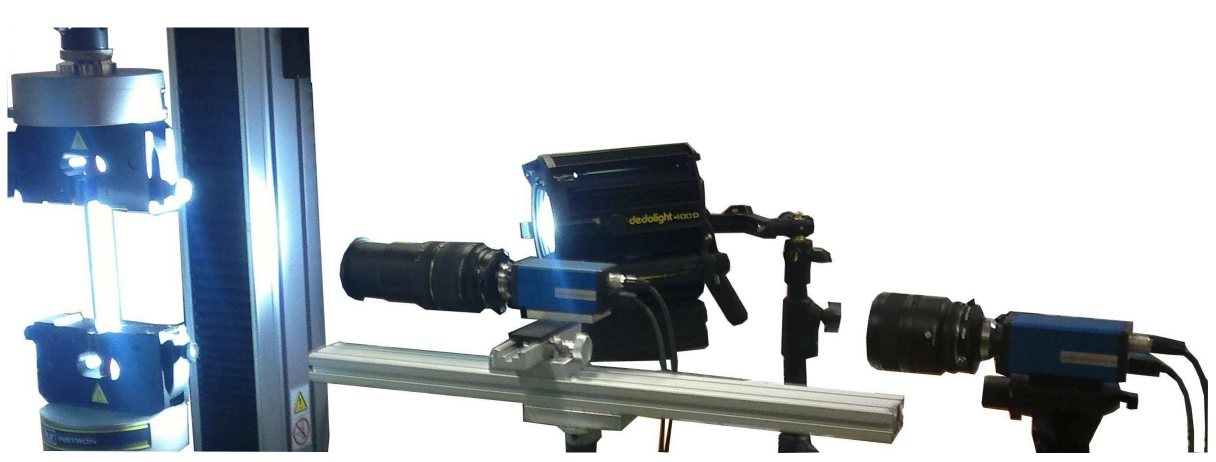

Fig. 8 Experimental setup. The two digital cameras are facing the specimen: their optical axis is perpendicular to the laminate plane. Here, a translation stage is used to retract the nearfied camera in order to capture the farfield image.

Two CCD cameras (AVT Dolphin F-145B) have been used to capture these images (definition: $1392 \times 1040$ pixels, 8-bit). Assuming that the specimen undergoes in-plane deformations, both the optical axes are set to be perpendicular to the laminate plane. The first camera takes pictures which cover approximately the whole gauge region, while the second concentrates over a smaller area located around the hole. The choice of the location of this nearfield region is justified in section 4.2. Here the ratio between the two optical resolutions is around 5. The nearfield camera, mounted on a translation stage, is then retracted to take a picture of the farfield region, see Figure 8.

A black and white speckle is sprayed on top of the surface in order to provide a random texture suited for the DIC. In practice, the speckle was intentionally made finer in the nearfield region [2], see Figure 7.

As expected in such a situation, the global load / displacement response is linear elastic. A typical simulation mesh is used in the FE-DIC to measure the displacement field at both scales. In practice, the simulation mesh is adjusted on the farfield image (the diameter of the hole and the width of the coupon are measured, but the position of the hole is adjusted). On the contrary, the mesh is adjusted automatically on the reference nearfield image using the optimized homography operator computed from real images, as described in Section 2.2. The multiscale images and corresponding mesh positions are plotted in Figure 9. The corresponding measured FE-DIC displacement fields along the tensile direction are presented 


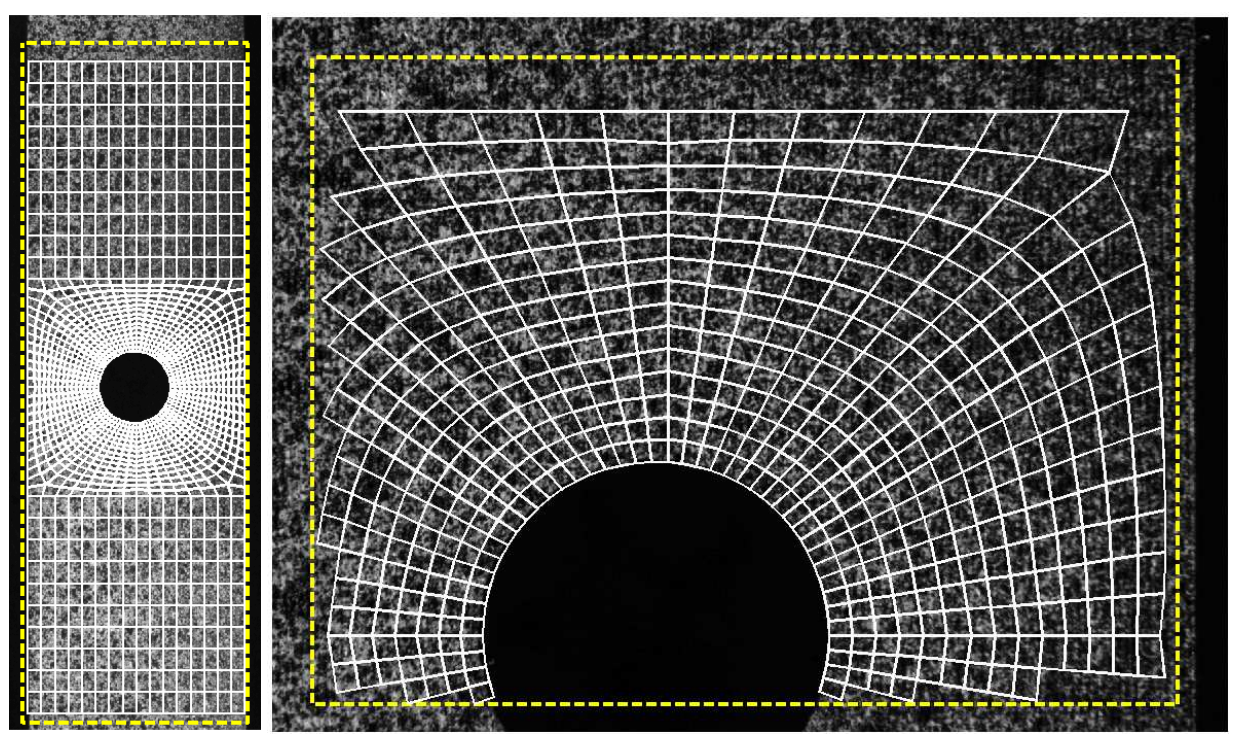

Fig. 9 Multiscale digital images with corresponding finite element mesh. The yellow lines correspond to the ROIs
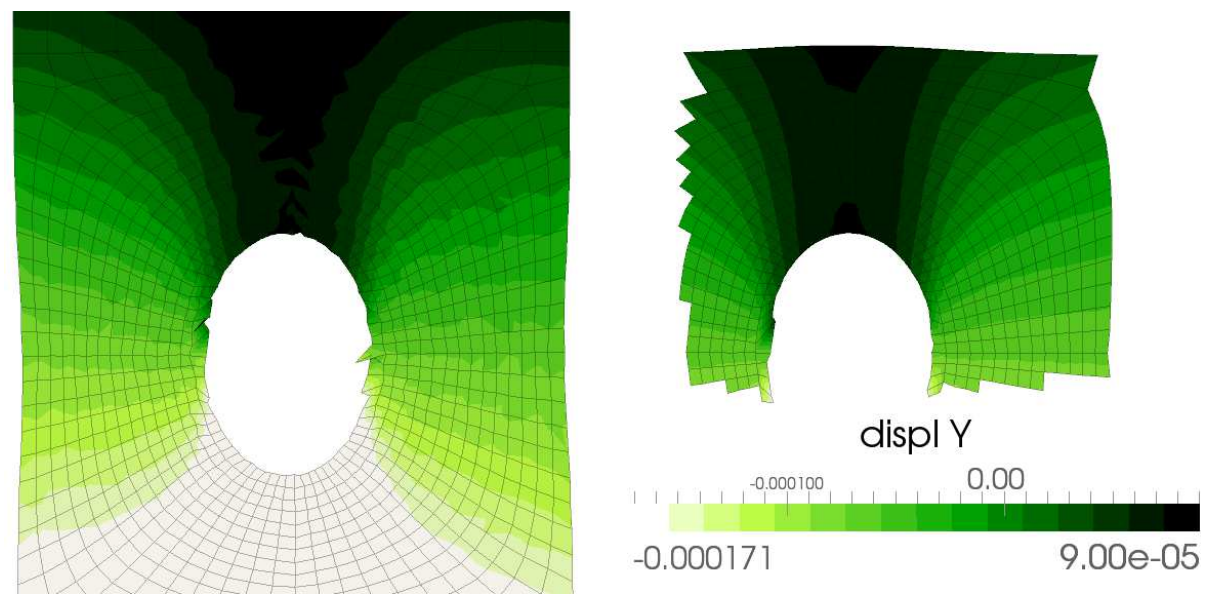

Fig. 10 Vertical $y$-component of the displacement field (in meter) measured by FE-DIC from farfield (left) and nearfield (right) images.

in Figure 10. It can be seen, even in the bare eye, that the displacement is more regular when using nearfield images. 

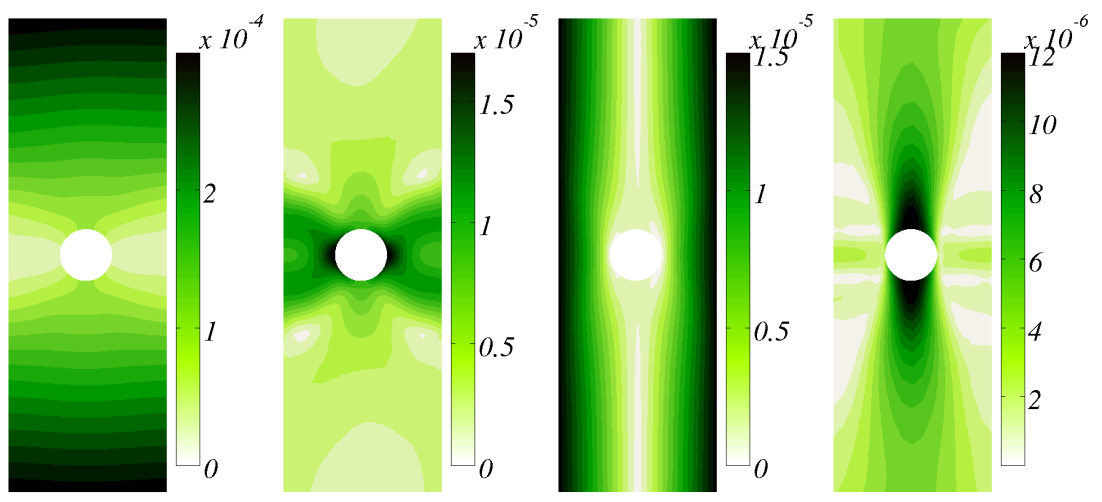

Fig. 11 Displacement sensitivity maps with respect to the four parameters to identify: (from left to right) $E_{l}, E_{t}, \nu_{l t}$ and $G_{l t}$

4.2 Choice of the nearfield region of interest

The identifiability of a constitutive parameter from full-field measurements obviously depends on the sensitivity of the field with respect to the sought parameter. The stacking sequence, the geometry and/or the loading play here a great role. In the following, the configuration of an open hole specimen subjected to a simple tensile test is evaluated.

The sensitivity of the displacement field $\mathbf{u}$ with respect to the constitutive parameter $p_{i}$ can be simply estimated from a couple of finite element computations using finite differences. An homogeneous orthotropic linear elastic is used to model the plate. To be representative, the applied boundary conditions are directly extrapolated from the FE-DIC farfield measurements. The constitutive parameters $\mathbf{p}$ are set to reference values. The latter were obtained classically by performing tensile tests on standard coupons (DIN EN ISO 527-4) [3,4,48]. Secondly, one computes the sensitivity $\delta \mathbf{u} / \delta p_{i}$. Figure 11 presents the sensitivity maps corresponding to the four in-plane elastic parameters $\left(E_{l}, E_{t}, \nu_{l t}, G_{l t}\right)$.

As expected in such a simple case, these maps highlight that the close vicinity of the hole is particularly relevant for identification purposes. The nearfield images will thus focus on this local area in order to get a higher displacement resolution (see Figure 9). Moreover the sensitivity analysis also exhibits that it will be much easier to identify the longitudinal Young modulus $E_{l}$ than the other parameters. 

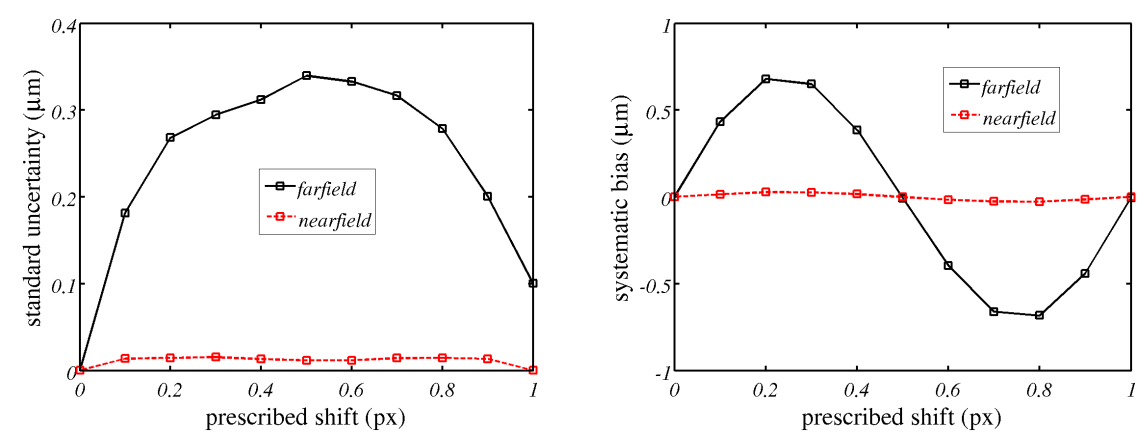

Fig. 12 Random (left) and systematic (right) errors (in $\mu \mathrm{m}$ ) as a function of the prescribed shift in pixel with the unstructured mesh.

In particular, a change of the transverse Young modulus $E_{t}$ will hardly affect the displacement field in a very narrow region.

\subsection{Error analysis of the real images}

An a priori performance analysis is performed on the real images, in order to assess the efficiency of the multiscale approach. A typical FE mesh is built for the simulation. It is irregular but structured and made of 4-noded bilinear elements whose size ranges gradually from $2.8 \mathrm{~mm}$ to $0.64 \mathrm{~mm}$ near the hole. Therefore the spatial resolution for the DIC displacement measurement varies from 33 to 145 (respectively 7 to 30 ) pixels in the nearfield (respectively farfield) image. In this case, the FE mesh is assumed to be optimized for the simulation. As a consequence, only the ultimate error is considered in this section. From the real reference images $\mathcal{I}_{0}^{f}$ and $\mathcal{I}_{0}^{n}$, two series of synthetic deformed images are generated by a subpixel shift in the Fourier space whose magnitude ranges between 0 and 1 pixel. A FEDIC measurement is then performed at both scales. The ultimate random error $\sigma^{u l t}$ and systematic error (bias) $\mu^{u l t}$ are thus computed from the discrepancy between measured and prescribed displacement fields, as described in section 3.2 for each value of the shift. The evolution of these two quantities is plotted in Figure 4.2 as a function of the shift magnitude in pixel. Note that for the farfield images, only measurement values inside the ROI corresponding to the nearfield images 
are considered in the error calculation. First, with the FE-DIC approach, typical bell-shaped and $S$-shaped curves are obtained for random and systematic errors respectively. This results is in good agreement with the litterature on subset based methods [42]. By using such a multiscale approach, the gain is hence double, since it takes advantage of both (a) the improvement of the image resolution (number of pixel $/ \mathrm{mm}$ ) and (b) the improvement of the DIC spatial resolution in pixel in the image (number of pixel/element width), for a given mesh. This corresponds respectively to (a) a vertical and (b) a horizontal translation between nearfield (red) and farfield (black) curves, in figure 6. Thanks to that, the ratio between nearfield and farfield uncertainties is more than one order of magnitude for an image resolution ratio of only 5 .

\section{Application to the identification of elastic properties}

Many techniques have been proposed to identify constitutive parameters from fullfield kinematical measurements [8]. Among them, the Virtual Fields Method [4951], the Equilibrium Gap Method [52-54] or the Finite Element Model Updating [6, $22,20,55]$ have for instance been used for composite materials. In the following, the latter technique is chosen. The objective here is to show how multiscale measurements can improve the performance of such an identification technique.

\subsection{Identification from multiscale measurements}

The Finite Element Model Updating (FEMU) method is a popular, intuitive and versatile identification technique $[21,8]$. It consists in updating a set of $p$ constitutive parameters $\mathbf{p}$ in a $\mathrm{FE}$ analysis in order to reduce, in the least squares sense, the distance $\mathbf{R}(\mathbf{p})$ between the measured and the simulated quantities.

$$
\mathbf{p}^{\star}=\underset{p \in \mathbb{R}^{p}}{\arg \min }\|\mathbf{R}(\mathbf{p})\|
$$

Different optimization techniques, norms $\|\cdot\|$ and cost functions may be used to exploit measured displacement fields $[21,8,6,22,11]$. For instance, in [6,22] strain fields are compared. In the following, we rather compare directly the displacement fields to avoid the amplification of the measurement noise linked to a numerical differentiation $[11,56]$. This calls for a strengthened emphasis on the boundary 
conditions. The identification of the elastic moduli requires moreover the minimization of the difference between the applied resultant load (measured by a load cell) and the simulated one. In this case, a hybrid residual vector $\mathbf{R}(\mathbf{p})$ is generally built as follows:

$$
\mathbf{p}^{\star}=\underset{p \in \mathbb{R}^{p}}{\arg \min } \sqrt{\left\|\mathbf{R}_{u}(\mathbf{p})\right\|^{2}+\left\|\mathbf{R}_{f}(\mathbf{p})\right\|^{2}}
$$

where the displacement and force residuals read:

$$
\begin{aligned}
& \mathbf{R}_{u}(\mathbf{p})=\frac{\mathbf{u}_{s}(\mathbf{p})-\mathbf{u}_{m}}{\left\|\mathbf{u}_{m}\right\|} \\
& \mathbf{R}_{f}(\mathbf{p})=\frac{F_{s}(\mathbf{p})-F_{m}}{F_{m}}
\end{aligned}
$$

where $\mathbf{u}$ denotes the displacement dof vector and $F$ the resultant force while .$m$ and.$_{s}$ stand respectively for the measured and the simulated quantities. A Levenberg-Marquardt algorithm is usually used to solve the minimization problem (19). Instead of using this $L^{2}$-norm, one could alternatively solve a weighted least squares problem. In particular, the FE-DIC correlation matrix $\mathbf{M}$ is related to the inverse of the covariance matrix for the degrees of freedom [17]. The use of $\mathbf{M}$ allows thus for a convenient weighting of the degrees of freedom [20,55]:

$$
\mathbf{p}^{\star}=\underset{p \in \mathbb{R}^{p}}{\arg \min } \sqrt{\left\|\mathbf{R}_{u}(\mathbf{p})\right\|_{M}^{2}+\left\|\mathbf{R}_{f}(\mathbf{p})\right\|_{2}^{2}}
$$

where the M-norm is defined as $\left\|\mathbf{R}_{u}\right\|_{M}^{2}=\mathbf{R}_{u}^{\top} \mathbf{M} \mathbf{R}_{u}$. It should be noted that the matrix $\mathbf{M}$ is symmetric and positive by construction. When invertible, it can be decomposed using the Cholesky decomposition as follows $\mathbf{M}=\mathbf{L L}^{\top}$. Thus the $M$-norm of $\mathbf{R}_{u}$ can be written using the $L^{2}$ norm as $\left\|\mathbf{R}_{u}\right\|_{M}=\left\|\mathbf{L}^{\top} \mathbf{R}_{u}\right\|_{2}$ which makes it compatible with a standard implementation of the Levenberg-Marquardt algorithm.

A simple standard monoscale FEMU approach is first applied. It exploits as usual exclusively the farfield FE-DIC measurements. On the one hand, the displacement field is measured using FE-DIC. On the other hand, a FE simulation if performed. The plate is meshed with the same mesh (optimized for simulation) as in section 4.3 (see Figure 9). As mentioned earlier, the simulated displacement field strongly depends on the chosen boundary conditions. To minimise the impact of this part of the modelling, the measured displacements on the nodes of the upper and lower boundaries are imposed as Dirichlet boundary conditions in 
the simulation. The reaction force $F$ can then be computed for the current set of constitutive parameters. As mentioned in section 3.2, the use of a locally refined mesh is relevant for the simulation. Nevertheless, it has to be coarse enough to measure accurate FE-DIC displacements. Indeed, a displacement field corrupted by large uncertainties will obviously yield large uncertainties on the identified parameters [20].

To avoid such a delicate compromise, a multiscale FEMU approach is thus developed. Like previously, farfield measurements are used to define Dirichlet boundary conditions in the FE simulation. They are indeed mandatory to compute the reaction $F_{s}(\mathbf{p})$. However, the FEMU now takes advantage of nearfield FE-DIC measurements. Once the simulation mesh adjusted on the reference nearfield image following the multiscale registration technique (see Section 2.2), one measures better resolved nodal displacements $\left.\mathbf{u}_{m}\right|_{\text {near }}$ in a restricted region where it is particularly sensitive to the sought parameters (see Section 4.2). As a first step, in this multiscale approach of FEMU, only those nearfield measurements are compared to the simulated displacements. The residual vector $\mathbf{R}_{u}(\mathbf{p})$ turns to:

$$
\mathbf{R}_{u}(\mathbf{p})=\frac{\mathbf{u}_{s}(\mathbf{p})-\left.\mathbf{u}_{m}\right|_{\text {near }}}{\left\|\left.\mathbf{u}_{m}\right|_{\text {near }}\right\|}
$$

Finally, a flowchart of the principal steps in the multiscale measurement and identification algorithm is provided in Figure 13. The strategy (global DIC measurements, optimizations, homography, image synthesis...) has been implemented in the Matlab environment. It takes less than 3 minutes to perform the overall multiscale identification strategy on a laptop with an Intel@ Core $^{\mathrm{TM}}$ i5 $2.53 \mathrm{GHz}$ CPU and 8Go memory.

\subsection{A priori analysis of the identification robustness}

In this section, the uncertainties associated to the identified parameters from synthetic images are compared. At each scale, an image is generated by warping the real reference images (Figure 9) with a displacement corresponding to the solution of the FE model with a known set of parameters denoted $\mathbf{p}_{\text {ref }}$. Both identification procedures are then applied to these two image pairs. To compare the approaches, the relative error between the reference $\mathbf{p}_{r e f}$ and the identified parameters $\mathbf{p}^{\star}$ 


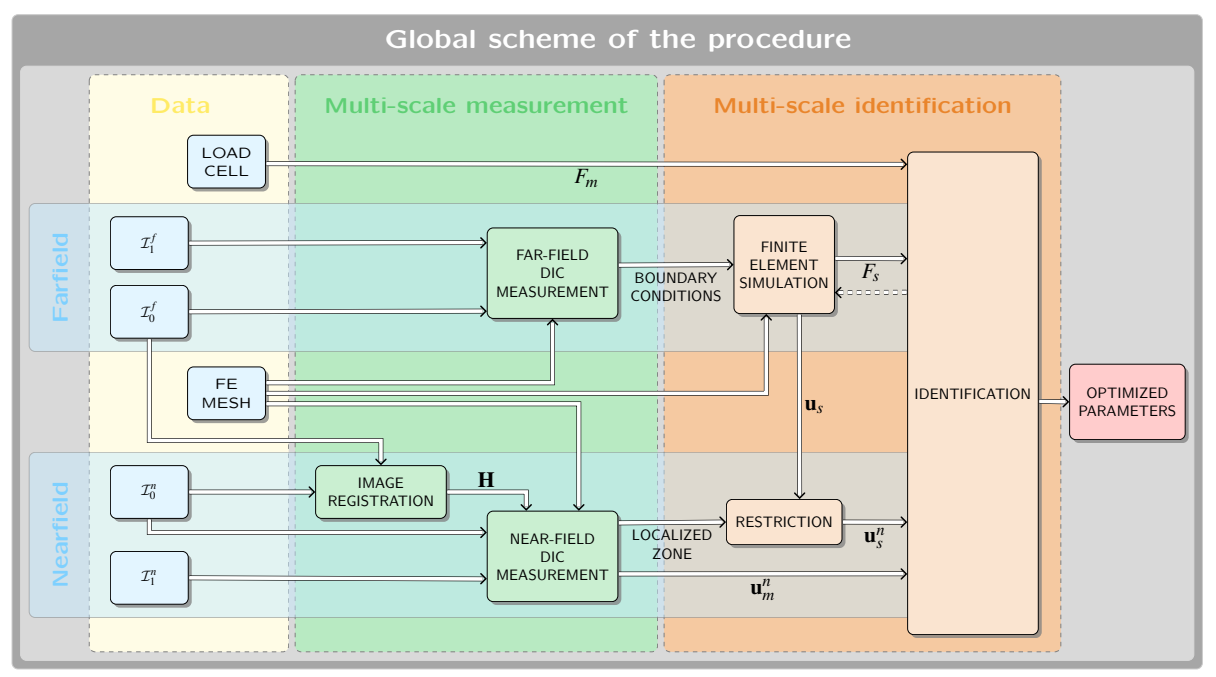

Fig. 13 Flowchart of the multiscale procedure

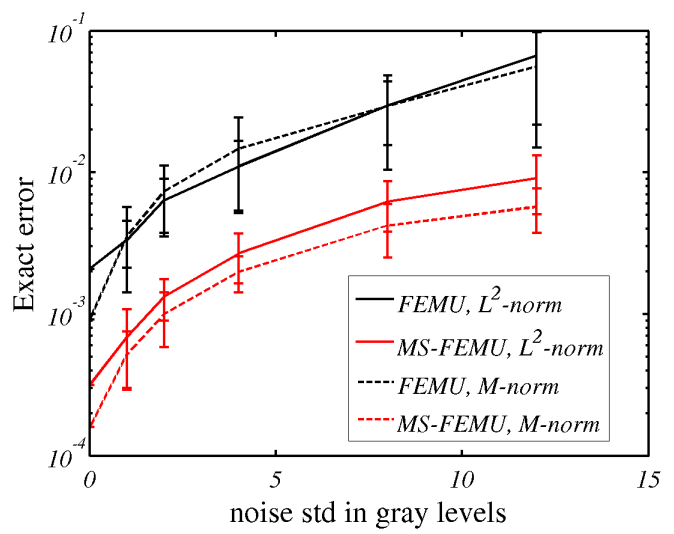

Fig. 14 Relative error $e(\mathbf{p})$ of the identified parameters as a function of the image noise for the standard single-camera identification technique (FEMU) and the proposed multiscale identification method (MS-FEMU) with the cost function expressed in the $L^{2}$-norm and $M$ norm. (note that the errorbars are also in logscale)

is quantified by $e\left(\mathbf{p}^{\star}\right)=\left\|\mathbf{p}_{r e f}-\mathbf{p}^{\star}\right\| /\left\|\mathbf{p}_{r e f}\right\|$. The standard and multiscale approaches give respectively $e\left(\mathbf{p}^{\star}\right)=2.08 \times 10^{-3}$ and $e\left(\mathbf{p}^{\star}\right)=3.15 \times 10^{-4}$. Thus the identification accuracy is improved by almost one order of magnitude on $e(\mathbf{p})$ with only a ratio of 5 between the image resolutions, in the proposed multiscale method. 
In addition, in order to further compare the robustness of the methods, the impact of the image noise is evaluated. A Gaussian noise, with zero mean and a standard deviation ranging from one to twelve gray levels, is added to the farfield and the nearfied images. For each level of noise, both the identification methods are run for 20 random samples. The evolution of the standard and multiscale identification errors are plotted in Figure 14 as a function of the image noise. It can be seen, that the proposed multiscale procedure reduces also the sensitivity of the identification with respect to image noise roughly by one order of magnitude. Finally, the use of the $M$-norm helps to further improve the identification accuracy $\left(e\left(\mathbf{p}^{\star}\right)=1.58 \times 10^{-4}\right.$ compared to $3.15 \times 10^{-4}$ previously $)$ and the noise robustness of the identified process.

\subsection{Identification from a true experimental image sequence}

The ten pairs of images recorded during the tensile test (see Section 4) are used to measure both farfield and nearfield displacement fields. The FEMU procedure is initialized using the elastic parameters identified classically (reference values): $E_{l}=$ $21.53 \mathrm{GPa}, E_{l}=20.59 \mathrm{GPa}, \nu_{l t}=0.15$ et $G_{l t}=3.54 \mathrm{GPa}[57]$. Figure 15 shows the evolution of the parameters identified with both standard and multiscale FEMU methods, at the nine last load steps.

As envisioned from the sensitivity analysis (Figure 11), the identification results demonstrate that the longitudinal modulus can correctly be estimated from both FEMU analyses. At the first loading steps, the multiscale approach produces more realistic values for all the parameters. Moreover, except for the transverse modulus $E_{t}$, the values of the parameters are close to the reference ones. It is worth remembering that the sensitivity of the displacement field with respect to this parameter is lower than for the other parameters, and that the highest sensitivities are restricted to a very small area. It is therefore logical that both approaches fail to provide relevant results for this parameter. On the other hand, the evolution of the identified parameters as a function of the loading step is much more regular with MS-FEMU than with standard FEMU, in particular at the first steps, where the signal to noise ratio is bad. This is in accordance with the uncertainty analysis of Figure 14. 

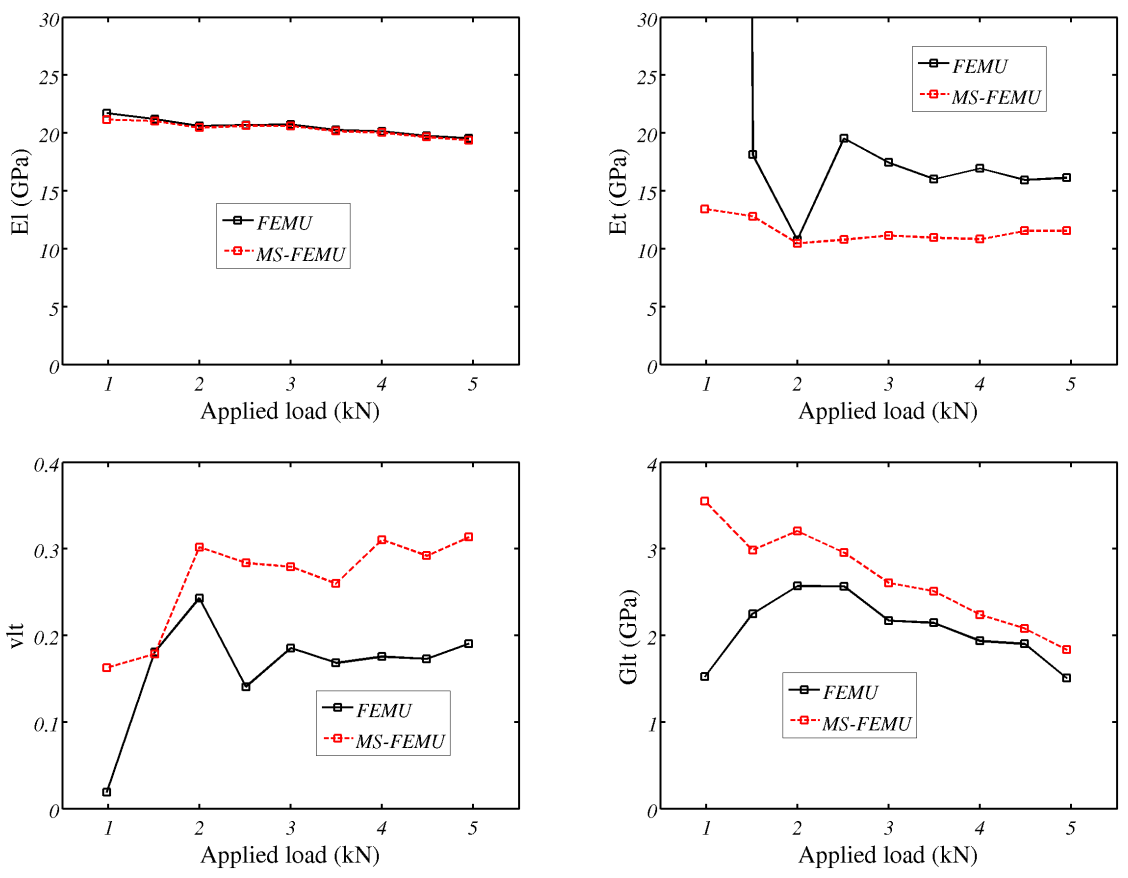

Fig. 15 Identified parameters from different loading magnitude. From top left to bottom right: $E_{t} ; E_{l} ; v_{l t} ; G_{l t}$ standard FEMU (solid line) and MS-FEMU (dashed line)

Paradoxically, the identification of the elastic properties of a composite laminate is quite a tricky problem. In order to stay in the elastic domain (including in the hole vicinity), the loading must be sufficiently low, which leads to small strain levels. The resulting signal to noise ratio makes standard FEMU fail to identify accurately elastic parameters. Conservely, high levels of loading, inevitably lead to material degradations (at least locally) which invalidate the elastic assumption. In this regard, the proposed multiscale identification technique is a good alternative since is proved to reduce significantly the noise sensitivity.

A complete analysis of the results reveals that the parameters seem to evolve significantly at a very early stage of the loading. The shear modulus $G_{l t}$ continuously decreases all along the tensile experiment. This could be related to the development of damage in the plate. The model considered herein (orthotropic linear elastic) is unable to model this phenomenon which is in addition most prominent in the nearfield region [58]. This may explain the reason why the 


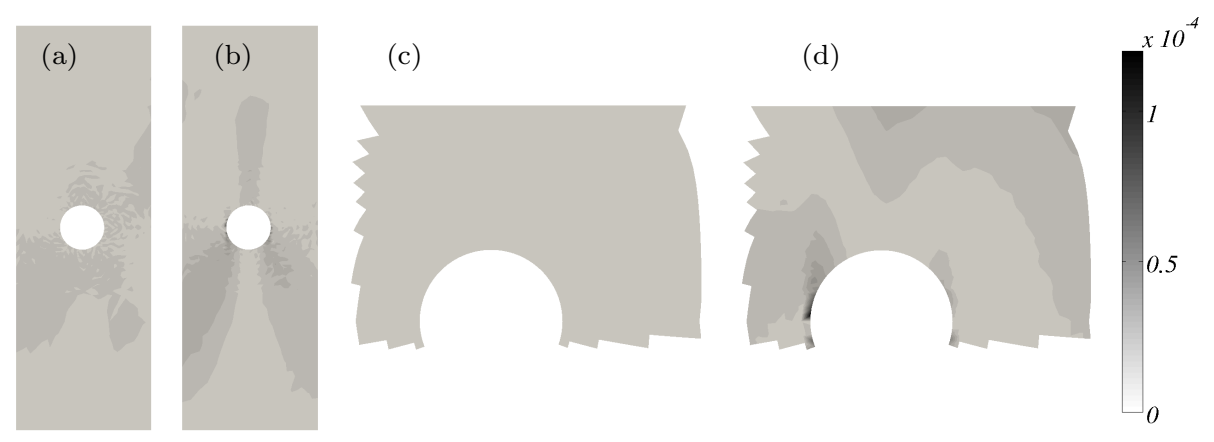

Fig. 16 Discrepancy maps of the vertical component of the displacement between measurement and simulation with the identified parameters for two extreme load amplitudes, $2 \mathrm{kN}$ (a and $\mathrm{c}$ ) and $5 \mathrm{kN}$ (b and d) for both the standard single-camera (a and b) and the proposed multiscale (c and d) FEMU.

parameters identified from the mono and the multiscale approaches can not be compared for the higher loading steps.

The difference between measured displacements $\mathbf{u}_{m}$ and the displacements $\mathbf{u}_{s}\left(\mathbf{p}^{\star}\right)$ simulated with the optimized parameters $\mathbf{p}^{\star}$ does give an interesting indicator of the relevance of the elastic assumption. Figure 16 shows the corresponding discrepancy maps for the set of parameters identified with the multiscale approach at both scales and at two distinct loading steps $(2 \mathrm{kN}$ and $5 \mathrm{kN})$. Discrepancies are present but hardly visible at $2 \mathrm{kN}$, but they are significant at $5 \mathrm{kN}$. Those maps simply confirm that the chosen model is (obviously) not able to describe the observed behavior throughout the tensile test, particularly in the vicinity of the hole where damage is known to localize.

\section{Conclusions}

Connection between simulation and full-field measurement was originally a critical task. With the advent of finite element based digital image correlation methods [16-18], it is now possible to bridge efficiently both of them with a common language: a finite element mesh $[20,55]$. However, choosing an appropriate mesh and / or spatial resolution may be quite tricky because of the spatial resolution 
/ uncertainty compromise $[12,42]$. In addition, this choice is also constrained by hardwamultiscalere limitations. Moreover, in the context of identification, the spatial resolution is often limited because the field of view generally needs to include the boundaries of the specimen [10]. At the same time, a high spatial resolution is required in the regions where the displacement is sensitive to the parameter to identify. And in some cases (which is the case of the real experiment here), these region are very small. All these remarks led us to devise both a new DIC methodology and an associated FEMU technique able to take the best of images taken at two different resolutions. Thus, in this paper, (a) a dedicated DIC method was proposed for the automatic and accurate registration of the farfield image in the nearfield image and (b) an hybrid multiscale cost function was used in the FEMU technique. Finally, to assess the effectiveness of the proposed multiscale approach, multi-resolution speckle pattern images were synthetized from a mechanical analytical field in order to simulate the whole chain from acquisition to the identification of elastic properties.

The results show that the proposed multiscale method significantly improves both measured displacements and identified parameters. It is shown that even with a ratio of 5 between the image resolutions, the measurement and identification uncertainties can be reduced by one order of magnitude which is one of the most interesting ouput of the study. Not only the uncertainties are reduced, but it is shown that the proposed method is also more robust with respect to image noise by approximately one order of magnitude. This noise robustness can be further reduced by using a weighted $\mathbf{M}$-norm as in [20].

Besides the case of more than two cameras or larger resolution ratios, there is a large number of work prospects, such as using other enhanced identification methods $[20,10]$, extension to stereo-DIC which is of great interest for more complex structures $[9,23]$. It may also avoid the movements of the nearfield camera between two shots. Ultimately, such multiscale methods will make sense when trying to identify multiscale simulation models including local non-linearities, like for instance damage $[59,60]$.

Acknowledgements The support of the "Agence Nationale de la Recherche" under grant VERTEX ANR-12-RMNP-0001-05 is gratefully acknowledged. 


\section{References}

1. M. Grédiac, F. Hild (eds.), Full-Field Measurements and Identification in Solid Mechanics (Wiley, 2012). ISBN 9781848212947

2. M. Sutton, J.J. Orteu, H. Schreier, Image correlation for shape, motion and deformation measurements: Basic Concepts, Theory and Applications (Springer, New York, NY (USA), 2009)

3. DIN EN ISO 527-4, Determination of the tensile properties of plastics, moulded materials, extruded materials and fibre reinforced plastic laminates (1997)

4. ASTM D3039-00, Standard test method for tensile properties of polymer matrix composite materials, vol. 15 (2000)

5. P. Meurs, P. Schreurs, T. Peijs, H. Meijer, Composites Part A: Applied Science and Manufacturing $\mathbf{2 7}(9), 781$ (1996)

6. J. Molimard, R. Riche, A. Vautrin, J. Lee, Experimental Mechanics 45(5), 404 (2005)

7. L. Bruno, G. Felice, L. Pagnotta, A. Poggialini, G. Stigliano, International Journal of Solids and Structures 45, 908 (2008)

8. S. Avril, M. Bonnet, A.S. Bretelle, M. Grédiac, F. Hild, P. Ienny, F. Latourte, D. Lemosse, S. Pagano, E. Pagnacco, F. Pierron, Experimental Mechanics 48(4), 381 (2008)

9. P. Sztefek, R. Olsson, Composites Part A: Applied Science and Manufacturing 39(8), 1282 (2008)

10. J. Réthoré, Muhibullah, T. Elguedj, M. Coret, P. Chaudet, A. Combescure, Int. J. Solids and Structures 50, 73 (2013)

11. M.B. Azzouna, P. Feissel, P. Villon, Measurement Science and Technology 24(5), 055603 (2013)

12. M. Bornert, F. Brémand, P. Doumalin, J.C. Dupré, M. Fazzini, M. Grédiac, F. Hild, S. Mistou, J. Molimard, J.J. Orteu, L. Robert, Y. Surrel, P. Vacher, B. Wattrisse, Experimental Mechanics 49(3), 353 (2009)

13. F. Hild, S. Roux, Experimental Mechanics 52(9), 1503 (2012)

14. M. Sutton, W. Wolters, W. Peters, W. Ranson, S. McNeill, Image and Vision Computing $\mathbf{1}(3), 133(1983)$

15. H. Bruck, S. McNeill, M. Sutton, W. Peters, Experimental Mechanics 29, 261 (1989)

16. Y. Sun, J. Pang, C.K. Wong, F. Su, Applied Optics 44(34), 7357 (2005)

17. G. Besnard, F. Hild, S. Roux, Experimental Mechanics 46(6), 789 (2006)

18. J. Fehrenbach, M. Masmoudi, Comptes Rendus Mathématique 346(9-10), 593 (2008)

19. Y.Q. Wang, M.A. Sutton, H.A. Bruck, H.W. Schreier, Strain 45(2), 160 (2009)

20. H. Leclerc, J.N. Périé, S. Roux, F. Hild, Lectures Notes in Computer Sciences 5496, 161 (2009)

21. K.T. Kavanagh, R.W. Clough, International Journal of Solids and Structures 7, 11 (1971)

22. D. Lecompte, A. Smits, H. Sol, J. Vantomme, D.V. Hemelrijck, International Journal of Solids and Structures 44(5), 1643 (2007)

23. P. Sztefek, R. Olsson, Composites Part A: Applied Science and Manufacturing 40(3), 260 (2009) 
24. B. Lucas, T. Kanade, in Proceedings of Imaging Understanding Workshop (1981), pp. $121-130$

25. B. Horn, G. Schunck, Artificial Intelligence 17, 185 (1981)

26. S. Roux, F. Hild, Y. Berthaud, Applied Optics 41(108-115) (2002)

27. F. Mortazavi, M. Levesque, I. Villemure, Strain 49(3), 233 (2013)

28. P. Cheng, M. Sutton, H. Schreier, S.R. McNeill, Experimental mechanics 42(3), 344 (2002)

29. J. Réthoré, T. Elguedj, P. Simon, M. Coret, Experimental Mechanics 50, 1099 (2010)

30. J.C. Passieux, J.N. Périé, International Journal for Numerical Methods in Engineering $\mathbf{9 2}(6), 531(2012)$

31. S. Roux, F. Hild, Int J. Fract 140, 141 (2006)

32. F. Hild, S. Roux, N. Guerrero, M.E. Marante, J. Flórez-López, European Journal of Mechanics - A/Solids 30(1), 1 (2011)

33. H. Leclerc, J.N. Périé, S. Roux, F. Hild, Experimental Mechanics 51(4), 479 (2011)

34. H. Bay, A. Ess, T. Tuytelaars, L.V. Gool, Computer Vision and Image Understanding $\mathbf{1 1 0}(3), 346(2008)$

35. J. Matas, O. Chum, M. Urban, T. Pajdla, Image and Vision Computing 22(10), 761 (2004)

36. D.G. Lowe, International Journal of Computer Vision 60(2), 91 (2004)

37. J.J. Orteu, D. Garcia, L. Robert, F. Bugarin, in Speckle'06 International Conference, vol. 6341 (2006), vol. 6341, p. http://dx.doi.org/10.1117/12.695280

38. K. Perlin, in SIGGRAPH'85 international conference, San Francisco, California, USA (1985), pp. $1643-1656$

39. P. Lava, S. Cooreman, S. Coppieters, M. De Strycker, D. Debruyne, Optics and Lasers in Engineering 47, 747 (2009)

40. H. Schreier, J. Braasch, M. Sutton, Optical Engineering 39(11), 2915 (2000)

41. S. Lekhnitskii, S.W. Tsai, T. Cheron, Anisotropic Plates. (Gordon and Breach, New York, USA, 1968)

42. F. Amiot, M. Bornert, P. Doumalin, J.C. Dupré, M. Fazzini, J.J. Orteu, C. Poilâne, L. Robert, R. Rotinat, E. Toussaint, B. Wattrisse, J.S. Wienin, Strain 49(6), 483 (2013)

43. C. Geuzaine, J.F. Remacle, International Journal for Numerical Methods in Engineering 79(11), 1309 (2009)

44. P. Reu, T. Miller, The Journal of Strain Analysis for Engineering Design 43(8), 673 (2008)

45. W. Wang, J.E. Mottershead, A. Ihle, T. Siebert, H.R. Schubach, Journal of Sound and Vibration 330(8), 1599 (2011)

46. G. Besnard, H. Leclerc, S. Roux, F. Hild, Journal of Strain Analysis for Engineering Design $\mathbf{4 7}(4), 214(2012)$

47. J. Gago, D. Kelly, O. Zienkiewicz, I. Babŭska, International Journal for Numerical Methods in Engineering 19, 1621 (1983)

48. M. Bizeul, C. Bouvet, J.J. Barrau, R. Cuenca, International Journal of Fatigue 32(1), 60 (2010)

49. H. Chalal, F. Meraghni, F. Pierron, M. Grédiac, Composites Part A: Applied Science and Manufacturing 35(78), 841 (2004) 
50. M. Grédiac, F. Pierron, S. Avril, E. Toussaint, Strain 42(4), 233 (2006)

51. F. Pierron, G. Vert, R. Burguete, S. Avril, R. Rotinat, M.R. Wisnom, Strain 43(3), 250 (2007)

52. S. Roux, F. Hild, Experimental Mechanics 48(4), 495 (2008)

53. L. Crouzeix, J.N. Périé, F. Collombet, B. Douchin, Composites Part A: Applied Science and Manufacturing 40(11), 1732 (2009)

54. M. Ben Azzouna, J.N. Périé, J.M. Guimard, F. Hild, S. Roux, International Journal of Damage Mechanics 20(8), 1130 (2011)

55. R. Gras, H. Leclerc, S. Roux, S. Otin, J. Schneider, J.N. Pri, Experimental Mechanics $\mathbf{5 3}(5), 719(2013)$

56. W. Wang, J.E. Mottershead, C.M. Sebastian, E.A. Patterson, International Journal of Solids and Structures 48(11-12), 1644 (2011)

57. M. Bizeul, C. Bouvet, J. Barrau, R. Cuenca, Composites Science and Technology 71(3), 297 (2011)

58. S. Hallett, B. Green, W.G. Jiang, K. Cheung, M. Wisnom, International Journal of Fracture 158(2), 169 (2009)

59. M. Geers, R. de Borst, T. Peijs, Composites Science and Technology 59(10), 1569 (1999)

60. B. Shen, G. Paulino, Experimental Mechanics 51(2), 143 (2011) 